P07.10 EVALUATION OF THE NEW BD MAX GC REAL TIME PCR ASSAY, ANALYTICALLY AND AS A SUPPLEMENTARY TEST TO THE BD PROBETEC GC QX AMPLIFIED DNA ASSAY, FOR MOLECULAR DETECTION OF NEISSERIA GONORRHOEAE

${ }^{1} \mathrm{D}$ Golparian*, ${ }^{2} \mathrm{~S}$ Boräng, ${ }^{1} \mathrm{M}$ Sundqvist, ${ }^{1} \mathrm{M}$ Unemo. ${ }^{1} \mathrm{WHO}$ Collaborating Centre for Gonorrhoea and Other Sexually Transmitted Infections, Swedish Reference Laboratory for Pathogenic Neisseria, Department of Laboratory Medicine, Microbiology, Faculty of Medicine and Health, Örebro University, Örebro, Sweden; ${ }^{2}$ Department of Clinical Microbiology, Karolinska University Hospital, Huddinge, Sweden

\subsection{6/sextrans-2015-052270.326}

Introduction The BD ProbeTec GC Qx Amplified DNA assay (Becton, Dickinson and Company) is used on the BD Viper System to detect Neisseria gonorrhoeae. However, suboptimal specificity and cross-reaction with commensal Neisseria species have been described. Recently, the BD Max GC real time (rt) PCR assay was developed for the BD Max System (BD) as a supplementary test.

Methods We evaluated the performance of the new BD Max GC rt PCR assay by examining clinical specimens positive in the BD ProbeTec GC Qx Amplified DNA assay during July-October 2014 among 23815 screening or clinical patients (14846 females and 8969 males) as well as samples spiked with isolates of gonococci $(\mathrm{n}=189)$, non-gonococcal Neisseria species $(\mathrm{n}=261)$ and other closely related bacteria $(n=10)$.

Results Of 23815 patients tested with the BD ProbeTec GC Qx Amplified DNA assay, 85 (0.6\%) females and 259 (2.9\%) males were positive. Of these 344 positive specimens, 322 were tested with BD Max GC rt PCR assay. Sixty-nine (21\%) of these samples were negative in BD Max GC rt PCR assay, a gonococcal dual target PCR and in the APTIMA Combo 2 (Hologic). These 69 specimens were obtained from pharynx $(50 \%$ of all screening positive pharyngeal specimens), urine (33\%), vagina (11.4\%), rectum (4.3\%), and cervix (1.4\%). In the analytical evaluation of the BD Max GC rt PCR assay, all gonococcal isolates were positive and all but one ( $N$. cinerea) of the non-gonococcal isolates (99.4\%) were negative. The $N$. cinerea isolate also cross-reacted in the BD ProbeTec GC Qx Amplified DNA assay.

Conclusion The BD ProbeTec GC Qx Amplified DNA assay had a suboptimal specificity for both urogenital and extragenital clinical specimens. The new BD Max GC rt PCR assay showed a high clinical and analytical sensitivity and specificity, and might also be used for initial detection of N. gonorrhoeae.

Disclosure of interest statement We are grateful to BD Diagnostics for providing the BD Max GC real time PCR tests. The present work was funded by grants from the Örebro County Council Research Committee and the Foundation for Medical Research at Örebro University Hospital, Sweden.

\section{P07.11 WHOLE GENOME SEQUENCING CHARACTERISATION OF A UNIQUE NEISSERIA STRAIN GENERATING A POSITIVE RESULT WITH THE COBAS $\otimes$ CT/NG TEST}

C Honisch*, S Cayabyab Hibbard, R Mehta, H Phan, S Sunkara, J Osiecki, M Lewinski, C Fillmore. Roche Molecular Systems, Inc., Pleasanton, CA, USA

10.1136/sextrans-2015-052270.327

Introduction Neisseria gonorrhoeae (NG) cause sexually transmitted bacterial infections. The Roche cobas ${ }^{\circledR} \mathrm{CT} / \mathrm{NG}$ Test is a qualitative Nucleic Acid Amplification test (NAAT) utilising a dualtarget approach to detect sequences within the direct repeat
(DR-9) region of the NG genome. To date, clinical performance data show excellent sensitivity and specificity for urogenital specimens. The test is validated for endocervical swabs, vaginal swabs and urine specimens.

Methods One clinical case report ${ }^{1}$ describes a positive test result for an oropharyngeal swab, an off-label specimen type for the cobas ${ }^{\circledR} \mathrm{CT} / \mathrm{NG}$ Test. This interesting Neisseria sample was subject to analysis as part of Roche's Global Surveillance Program. The isolate was tested by culture, phenotyping, whole cell MALDI-TOF MS and molecular methods such as $16 \mathrm{~S}$ rDNA based typing and whole genome sequencing.

Results Whole genome sequencing resulted in a complete genome assembly supporting phylogenetic analysis. Sequencing data assembled into a 2,882,113bp contig and genome closure, which identified the sample as a Neisseria commensal species clustering in the same clade with both N. sicca and N. macacae.

One partial assay specific DR-9 target region was identified within the genome of this commensal strain in the context of a phage like motif, which can facilitate genomic exchange of DNA fragments between $N$. gonorrhoeae and commensal strains. BLAST searches for the prophage/DR9 genetic signature of this newly characterised Neisseria strain revealed no matches to sequences in the public domain, suggesting that this strain is rare.

Based on the sequence information of the prophage/DR9 genetic signature oropharyngeal swab specimens determined to be positive by the cobas ${ }^{\circledR} \mathrm{CT} / \mathrm{NG}$ Test are currently being evaluated for the presence of this sequence motif.

Conclusion The cobas ${ }^{\circledR}$ CT/NG Test on the cobas 4800 system is a reliable molecular method for detection of Chlamydia and Gonorrhoeae from genital specimens.

Disclosure of interest Authors of the abstract are employees of Roche Molecular Systems.

\section{REFERENCE}

1 Upton A, Bromhead C, Whiley DM. Neisseria gonorrhoeae false-positive result obtained from a pharyngeal swab by using the Roche cobas 4800 CT/NG assay in New Zealand in 2012. J Clin Microbiol. 2013;51:1609-10

\section{P07.12 FACTORS INFLUENCING THE DETECTION OF NEISSERIA GONORRHOEAE FROM THE TONSILS AND POSTERIOR OROPHARYNX}

${ }^{1,2} \mathrm{M}$ Bissessor*, ${ }^{3,4} \mathrm{DM}$ Whiley, ${ }^{1} \mathrm{DM}$ Lee, ${ }^{1} \mathrm{AF}$ Snow, ${ }^{1,5} \mathrm{CK}$ Fairley, ${ }^{1,5} \mathrm{CS}$ Bradshaw, ${ }^{2}$ JS Hocking, ${ }^{6,7} \mathrm{M}$ Lahra, ${ }^{1} \mathrm{~J}$ Peel, ${ }^{1,5} \mathrm{MY}$ Chen. ${ }^{1}$ Melbourne Sexual Health Centre, Alfred Health, Melbourne, Victoria, Australia; ${ }^{2}$ Melbourne School of Population and Global Health, University of Melbourne, Melbourne, Victoria, Australia; ${ }^{3}$ The University of Queensland, St Lucia, Queensland 4072, Australia; ${ }^{4}$ Queensland Paediatric Infectious Diseases Laboratory, Queensland Children's Medical Research Institute, The University of Queensland; ${ }^{5}$ Central Clinical School, Monash University, Melbourne, Victoria, Australia; ${ }^{6}$ WHO Collaborating Centre for Sexually Transmitted Diseases, SEALS Microbiology, The Prince of Wales Hospital, Randwick, New South Wales 2031, Australia; ' University of New South Wales, Kensington, New South Wales 2052, Australia

\subsection{6/sextrans-2015-052270.328}

Background Limited data exists on the specific anatomical areas within the pharynx from which Neisseria gonorrhoeae can be detected. We examined factors influencing the detection of gonorrhoea from the pharynx.

Method Men who had sex with men (MSM) diagnosed with pharyngeal gonorrhoea by culture were recalled for repeat swabbing 7 days later: firstly from both tonsils then, using separate swabs, from the posterior oropharynx. These were tested for $N$. gonorrhoeae using culture and real-time PCR targeting the 